\title{
Aufmerksamkeitsstörung (ADS) - eine Diagnose, die weder Patienten noch Behandlern hilft
}

\author{
For and Against: Attention Deficit Syndrome (ADS) - A Diagnosis that Neither \\ Helps Patients nor Therapists
}

Bibliografie

DOI $10.1055 / \mathrm{s}-2006-940149$

Psychiat Prax 2007; 34: 4-6

(c) Georg Thieme Verlag KG

Stuttgart · New York .

ISSN 0303-4259

Korrespondenzadressen

Dr. med. Thomas Weniger

Gartenstraße 8

88239 Wangen

thweniger@aol.com

\section{Dr. med. Dipl.-Psych. Barbara Alm}

Zentralinstitut für Seelische Gesundheit, Klinik für Psychiatrie und Psychotherapie, 15 68159 Mannheim

barbara.alm@zi-mannheim.de

\section{Pro}

Gegen Ende der 50er-Jahre brach in der amerikanischen Stadt Seattle eine merkwürdige Epidemie aus: immer mehr Autofahrer stellten fest, dass ihre Windschutzscheiben von kleinen pocken- oder kraterähnlichen Kratzern übersäht waren. Hinsichtlich einer Erklärungstheorie dieses Phänomens, das in der Folge Präsident Eisenhower sogar zum Einsetzen einer Untersuchungskommission vor Ort veranlasste, zerfielen die Betroffenen in zwei Lager: die „Fallout“-Fraktion mutmaßte einen Zusammenhang mit kürzlich zurückliegenden russischen Atomtests, die „Asphalttheoretiker“ verdächtigten ursächlich frisch asphaltierte Autobahnen. Als die Männer des Eichamtes jegliche objektivierbare Zunahme zerkratzter Autoscheiben schließlich verneinen konnten, erwies sich, dass die Berichte über die Existenz pockennarbiger Autoscheiben in den Medien dazu geführt hatten, dass immer mehr Autobesitzer, anstatt wie bisher von innen durch ihre Frontscheiben zu sehen, diese mittels genauer Aufsicht untersucht hatten, indem sie sich von außen über ihre Scheiben beugten und sie auf kürzeste Entfernung anstarrten, wobei sich unter diesem Blickwinkel bislang unerkannte normale Abnützungsspuren erkennen ließen [1]. Die erhebliche Zunahme von als aufmerksamkeitsgestört diagnostizierten Patienten sowie hiermit einhergehend die Verordnungshäufigkeit von Psychostimulanzien in den vergangenen Jahren [2], zwingt zu einer distanziert kritischen Betrachtung dieses Phänomens. Unsicherheit vermag insbesondere der Umstand zu erzeugen, dass die ADS-Diagnose in keiner Weise auf diskret wahrnehmbaren Phänomenen fußt (wie dies z. B. bei einem Beinbruch der Fall ist, entweder gebrochen oder eben nicht), sondern auf der synthetischen Betrachtung einer quantitativ auffälligen Art und Weise, im Leben zu stehen. Hinzu kommt, dass dieses „pattern“ erst dann zum Problem wird, wenn es auf Umgebungs- und Er- wartungsbedingungen trifft, die damit nicht in Übereinstimmung zu bringen sind („not to fit“), was dieses einer ähnlichen Problematik unterwirft, wie sie auch dem Psychopathiebegriff zugrunde liegt, weil der Integrationsfähigkeit der Umgebung eine - vielleicht: entscheidende Wirkung in Hinsicht auf die Manifestation einer ADS-Problematik zukommt.

Eine um Entpathologisierung bemühte Sicht könnte „ADSler“ ganz allgemein gesprochen auch als Menschen verstehen, deren besondere neuronale Verdrahtung (oder Transmitterbesatz) zu einer Fähigkeit (und Neigung) zur Parallelverarbeitung von Informationen bei gleichzeitiger Filterschwäche führt. Bedingt dies einerseits die Gefahr einer Wahrnehmungsüberschwemmung („over-inclusement“), können Betroffene durch eine überdurchschnittliche Intelligenz und Leistungsfähigkeit einer Dekompensation oft erfolgreich entgegenarbeiten. Verglichen mit einem Computer würden ADSler also dazu neigen, mehrere Programme gleichzeitig laufen zu lassen, wobei ein rasch taktender Prozessor bei deren Bewältigung hilfreich ist. Da diese Arbeitsweise jedoch vergleichsweise viel Strom aus dem Akku zieht, muss dieser über den Dynamo Stimulation zeitnah aufgefüllt werden, was im Ergebnis zur bekannten Gradwanderung zwischen möglicher Über- bzw. Unterstimulation der Merkmalsträger führt.

Insbesondere die Unterforderung, verbunden mit Empfindungen von Leere oder Langeweile („verdünnter Schmerz“), erklärt die äußerlich erkennbare Neigung zu motorischer (Hibbeln, Ticks, Überbewegungen) oder pharmakologischer (Koffein, Nikotin, andere Drogen) Selbststimulation Betroffener sowie das zwanghaft anmutende Aufsuchen aller Arten von Außen- bzw. Fremdstimulation („Dopamin-Junkies“, „sensation-seeking-behavior" mit Wahl entsprechender beruflicher Tätigkeiten, aber auch Konfliktschürer, die provozieren und sich „zum Affen machen“). 
Treffen hierbei mögliche Fehlanpassungen wie Stimmungslabilität, Sucht, Dissozialität auf einen Behandler, der sich in der Pflicht fühlt, eine übergreifende Kategorisierung in ein griffiges Krankheitskonstrukt vorzunehmen, ist ein neuer ADSler geboren. Für den Betreffenden beinhaltet diese diagnostische Einordnung neben dem „Segen“ nunmehr spezialisierter professioneller Zuwendung einschließlich möglicher BTM-pflichtiger pharmakologischer Unterstützung jedoch einen hohen Preis, der im Einschwingen auf einen immensen identitätsbildenden Attraktor (ein Selbstkonzept mit Sogwirkung) besteht, dem neben entlastenden („ach deshalb komme ich im Beruf mit Vorgesetzten nicht zurecht“) auch aufwertende („auch Mozart und Einstein zählen ja angeblich zum Klub“) und durch äußere Verstärker (Wucht der Randgruppenidentität) gestützte Implikationen innewohnen und der sich in der Praxis als ausgesprochen schwer aufhebbar, ja geradezu „klebrig“, erweist.

So kann man sich einen Jetpiloten konstruieren, der gerade aufgrund seiner ADS-Besonderheiten im Cockpit über Jahre und Jahrzehnte seine überragenden Fähigkeiten ausspielen kann (dort bekommt er ja auch ausreichend Stimulationsinput!) und der dann, aufgrund eines Bandscheibenleidens zum Bodenpersonal versetzt anhand der ihn langweilenden Arbeit „plötzlich mit ADS dekompensiert“.

Ist es akzeptabel, sich einem generalisierenden Krankheitskonzept zu- und unterordnen zu lassen, das in Hinsicht auf seine „Verwirklichung“ von solchen Variablen abhängig ist? Oder gilt es nicht vielmehr, ADS-Eigenschaften im Einzelfall - ähnlich dem Zielen über Kimme und Korn beim Schießen - einem Abgleich mit den jeweiligen individuellen Lebens- und Beziehungsanforderungen der Einzelsituation zuzuführen und somit hochindividualisierte Lösungsansätze ohne übergreifendes Konstrukt vorzunehmen? Dies würde dann beispielhaft zu einer Aussage führen, wie sie mir seitens eines meiner Klienten vorgetragen wurde: „Im Hinblick auf meine Tätigkeit als Biologielehrer habe ich nach der zweiten Stunde ein ADS, wenn es mir aber gelingt, meinen Stundenplan so zu gestalten, dass ich zwischen Biologieund Sportunterricht hin und her wechseln kann, habe ich kein ADS“.

Solcher Sichtweise kann mit der derzeitigen diagnostischen und therapeutischen Situation nur unzureichend Rechnung getragen werden, nach welcher im Wunsch, zu einem objektiven Einoder Ausschluss zu gelangen, eine Zuweisung zur Dichotomie betroffen/nicht betroffen und damit mittelbar krank/gesund erfolgt, womit aus der Forderung nach einer Hochindividualisierung in der Praxis eher die Gefahr einer Hochinvalidisierung entsteht.

Weil das so ist und weil nach meiner persönlichen Erfahrung und Überzeugung die Vorteile dieses Vorgehens begonnen haben, hinter die Nachteile zurückzutreten, schlage ich vor, die Sicherheit eines als Krankheitsentität festlegbaren ADS aufzugeben, wie dies in Hinblick auf die Homosexualität in der Vergangenheit getan wurde. Um nochmals abschließend mit Watzlawick zu sprechen: damit waren auf einen Schlag Millionen von Menschen geheilt!

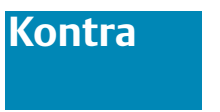

Die Wahrnehmung, Diagnostik und Therapie der Aufmerksamkeitsdefizit-/Hyperaktivitätsstörung (ADHS) bei Erwachsenen hat sich im vergangenen Jahrzehnt deutlich verändert. ADHS, gekennzeichnet durch situationsübergreifende Störungen der Aufmerksam- keit, der Impulskontrolle, motorische Hyperaktivität, Desorganisiertheit und Affektdysregulation wurde lange Zeit ausschließlich als eine Störung des Kindes- und Jugendalters gesehen. Stand der Wissenschaft durch Follow-up-Studien an Kindern ist heute, dass ADHS bei einem Teil der Betroffenen (ca. 50\%) auch bis in das Erwachsenenalter weiter bestehen kann. ADHS wird zunehmend verstanden als eine lebenslange Störung, die schwerwiegende Konsequenzen für die Betroffenen haben kann [3].

Bezüglich der Prävalenz im Erwachsenenalter ergab eine kürzlich in den USA durchgeführte epidemiologische Studie bei 3917 Personen im Alter von 18-44 Jahren eine Prävalenz von 4,4\% für ADHS im Erwachsenenalter [4]. Auch Querschnittsuntersuchungen zeigten an verschiedenen Populationen (Führerscheinbewerber, Studenten) Prävalenzen von über 4\%. ADHS hat für einen Teil der Erwachsenen schwerwiegende negative Konsequenzen. Nach bisher vorliegenden Studienergebnissen besteht bei 60-80\% der betroffenen Erwachsenen mindestens eine weitere psychische Störung, hauptsächlich affektive Störungen, Angststörungen und Substanzabhängigkeiten. Weniger gut untersucht sind Persönlichkeitsstörungen, wobei Studienergebnisse ein erhöhtes Risiko für dissoziale Persönlichkeitsstörungen bzw. delinquentes Verhalten konsistent belegen. Weitreichende psychosoziale Folgen, wie Schulabschlüsse, Ausbildungen und berufliche Tätigkeiten unterhalb des intellektuellen Begabungsniveaus, erhöhte Arbeitslosen- und Scheidungsrate, Partnerschaftskonflikte, Beeinträchtigung von Erziehungsfunktionen, Selbstwertstörungen und Beeinträchtigungen der Fahreignung wurden im Vergleich zu nicht betroffenen Kontrollprobanden in Untersuchungen wiederholt beschrieben [5].

2003 sind Leitlinien zur Diagnostik und Therapie der Erwachsenen-ADHS veröffentlicht worden, die Diagnosekriterien und soweit vorhanden - evidenzbasierte Therapieempfehlungen geben [6]. Ein multimodaler Ansatz, der den vorliegenden Symptomen, Funktionsstörungen und komorbiden Störungen entspricht und Psychopharmakotherapie und Psychotherapie kombiniert, wird favorisiert und - vielfach kritisiert.

Der Wissenschaftliche Beirat der Bundesärztekammer äußerte 2005, dass die Besorgnis über die Zunahme der Verordnung von Stimulanzien (hier Methylphenidat) unbegründet und eher Ausdruck einer Unterversorgung mit Medikamenten gewesen sei.

Kritikpunkte sind, dass 1. die Diagnose ADHS zu häufig gestellt, bzw. die Krankheit als solche gar nicht vorhanden sei, sondern als Erklärung für ein vielfaches Versagen der Umwelt oder der Personen selbst herhalten müsse, 2. die Diagnose die Betroffenen bezüglich ihrer psychosozialen Probleme in Passivität verharren lasse und 3. die Therapie mit Stimulanzien viel zu häufig erfolge, bzw. falsch sei.

\section{Diagnosestellung}

Die Diagnose ADHS ist eine klinische Diagnose, kein Test (biologische Marker, neuropsychologische Testbatterien oder funktionelle Bildgebung) kann die exakte klinische Untersuchung ersetzen. Die Diagnostik ist aufwändig und zeitintensiv, die Symptome müssen in ihrer Zeitstabilität erfasst werden, eine Fremdanamnese erhoben und weitere Erkrankungen, die die Symptome erklären könnten, ausgeschlossen werden. ADHS ist eine Ausschlussdiagnose.

Nur exaktes Arbeiten von Medizinern kann davor schützen, Fehldiagnosen zu stellen und den Betroffenen Therapien vorzuenthalten. Erfahrungsberichte von Patienten zeigen, dass die Diagnosestellung ihnen einen Kontext gab, Emotionen und Hand- 
lungen zu verstehen, aber auch ihre Ressourcen zu nutzen. Patienten berichten, dass sie ihre Kindheit so in Erinnerung haben, dass, was immer sie auch getan haben, nie gut genug war. Sie mussten sich immer anstrengen, Dinge anders machen, nur wie, habe ihnen keiner gesagt.

Das National Institute of Mental Health stimmte 1998 in einem Consensus Statement zu, dass verschiedene klinische Modelle für ADHS existieren und substanzielle Evidenz für die Validität der Störung bestehe und betont die negativen Langzeitfolgen, die aus nicht oder fehlender Diagnosestellung und Behandlung resultierten [7].

\section{Psychosoziale Beeinträchtigung}

Stellvertretend für diese Thematik soll der bisher wenig untersuchte Bereich der Elternschaft bei Erwachsenen mit ADHS diskutiert werden. ADHS-Mütter werden als weniger konsistent und ungeduldiger in ihrem Erziehungsverhalten beschrieben. In einer Studie von McGough, der Eltern von Kindern, die ADHS hatten $(n=152)$ und Eltern ohne ADHS $(n=283)$ untersuchte, zeigten sich bei den ADHS-Eltern mehr Substanzabhängigkeiten, depressive Störungen und Angststörungen, auch fand sich ein früherer Beginn der Depression. Diese Komorbidität, so interpretiert der Autor seine Ergebnisse, zeige nicht nur persönliches Leiden und Fehlanpassung der Betroffenen, reflektiert durch niedrigen Bildungsstand und geringen beruflichen Erfolg, sondern zeige auch den Einfluss auf die Familien, die durch die elterliche Psychopathologie belastet sind. Mc Gough spricht hier von ADHS als „family affair“ [8]. Dies ist ein weiteres Argument für die ADHS-Diagnosestellung, denn Information und Beratung entlasten und Prävention kann helfen, anders mit den psychosozialen Problemen umzugehen.

\section{Therapie}

Kritisiert wird die Medikalisierung von abweichenden Verhaltensweisen. Die Therapie mit Stimulanzien begünstige eine spätere Drogenabhängigkeit.

Wie oben dargestellt, ist die exakte Diagnosestellung die Voraussetzung dafür, um mit dem Patienten im Sinne eines „shared decision making“ Therapieoptionen zu besprechen, u.a., ab wann behandelt werden soll und ob der Patient Stimulanzien einnehmen möchte. Evidenz für die Wirksamkeit von Methylphenidat bei Erwachsenen mit ADHS liegt vor [9], Studien konnten auch belegen, dass durch Methylphenidat keine Abhängigkeit induziert wird, eine frühe Behandlung zeigte eine protektive Wirkung bezüglich Drogenkonsum bei Jugendlichen und jungen Erwachsenen [10]. In der Behandlung sollten auch Psychotherapien eine Rolle spielen. Allerdings liegen bisher nur wenige Studien zur Wirksamkeit von Psychotherapie vor. Die Freiburger ADHS-Arbeitsgruppe hat ein Manual für eine spezifische Gruppentherapie entwickelt und in einem kontrollierten klinischen Versuch an 76 Patienten mit guten Ergebnissen in Bezug auf die ADHS-Symptomatik durchgeführt [11].

\section{Fazit}

Die Diagnosestellung bei Erwachsenen mit ADHS ist notwendig und wichtig, weil die Störung im Erwachsenenalter nach vorliegenden Studien häufig ist und mit vielfältigen psychosozialen Beeinträchtigungen einhergeht, die in ätiologischem Zusam- menhang mit verschiedenen Vulnerabilitätsfaktoren zu sehen sind. Therapieziele sind Selbstmanagement und Selbstkontrolle der ADHS-Symptome. Nur durch die Diagnosestellung kann dem Betroffenen die Möglichkeit zur Auseinandersetzung und Veränderung seiner Erfahrungswelt gegeben werden. Entscheidend für neue Lernprozesse ist, dass alternative Erfahrungen gemacht werden, unabhängig davon, wie (durch Medikamente, Psychotherapie oder beides) diese gemacht werden. Mit der Diagnose kann der Patient sich viele Voraussetzungen schaffen, positive Erfahrungen zu machen, Ressourcen zu aktivieren. Und wir als Behandler sollten die Perspektivenvielfalt der ADHS-Störung kennen und die Bereitschaft haben, uns mit dem eigenen Handeln auseinanderzusetzen.

Eine Krankheit? Keine Krankheit? Die Auseinandersetzung um diese Frage ist in Gang und polarisiert. Aber was sagen die betroffenen Erwachsenen? Ein kürzlich veröffentlichtes Editorial im American Journal of Psychiatry plädiert dafür, die Wünsche des Patienten wieder mehr in den Mittelpunkt zu stellen. Wir messen und messen und vergessen, die Patienten zu fragen: Wie fühlen Sie sich? Dies als Aufforderung für uns alle [12]. Die gute Nachricht aus den verfügbaren Studien zum Thema ADHS ist die, dass ADHS behandelbar ist. Die ADHS-Forschung ist sehr aktiv. Wir wissen zunehmend mehr über die biologische Basis, beteiligte Gehirnstrukturen und bedeutsame Umwelteinflüsse, Gen-Umwelt-Interaktionen sind wahrscheinlich. Und je mehr wir wissen, umso genauer wird die Diagnostik und umso wirkungsvoller die Behandlung.

\section{Literatur}

1 Watzlawick P. „Wie wirklich ist die Wirklichkeit“. München: Piper, 1976: 84

2 Schubert I, Lehmkuhl G, Spengler A et al. Methylphenidat bei hyperkinetischen Störungen. Verordnungen in den 90er-Jahren. Dtsch Ärztebl 2001; 98: A 541 - 544

3 Biederman J. Attention-deficit/hyperactivity disorder: A selective overview. Biol Psychiatry 2005; 57: 1215-1220

4 Kessler RC, Adler L, Barkley R, Biederman J, Conners CK, Demler O, Faraone SV, Greenhill LL, Howes MJ, Seenik K, Spencer T, Ustun TB, Walters EE, Zaslavsky AM. The Prevalence and Correlates of Adult ADHD in the United States: Results From the National Comorbidity Survey Replication. Am J Psychiatry 2006; 163 (4): 716-723

5 Sobanski E, Alm B. Aufmerksamkeitsdefizit-/Hyperaktivitätsstörung (ADHS) bei Erwachsenen. Nervenarzt 2004; 75: 697-715

6 Ebert D, Krause J, Roth-Sackheimer C. ADHS im Erwachsenenalter Leitlinien auf der Basis eines Expertenkonsens mit Unterstützung der DGPPN. Nervenarzt 2003; 10: 939-946

7 Diagnosis and Treatment of Attention Deficit Hyperactivity Disorder. NIH Consens Statement 1998; 16 (2): 1 - 37

8 McGough JJ, Smalley SL, McCracken JT, May Yang MS, Del'Homme M, Lynn DE, Loo S. Psychiatric comorbidity in adult attention deficit hyperactivity disorder: findings from muliplex families. Am J Psychiatry 2005; 162: 1621 - 1627

9 Faraone SV, Spencer T, Aleardi M, Pagano C, Biederman J. Meta-analysis of methylphenidate for treating adult attention-deficit/hyperactivity disorder. J Clin Psychopharmacol 2004; 24: 24-29

10 Wilens TE, Faraone SV, Biederman J, Gunawardene S. Does stimulant therapie of attention-deficit/hyperactivity disorder beget later substance abuse? A meta-analytic review of the literature. Pediatrics 2003; 111: 179- 185

11 Hesslinger B, Philipsen A, Richter H, Ebert D. Psychotherapie der Aufmerksamkeitsdefizit-/Hyperaktivitätsstörung (ADHS) im Erwachsenenalter. Ein Arbeitsbuch. Göttingen: Hogrefe, 2004

12 Gabbard GO, Freedman R. Psychotherapy in the journal: What's missing? Am J Psychiatry 2006; 163: $182-184$ 